\title{
Hyperbolic Octonionic Proca-Maxwell Equations
}

\author{
Nuray Candemir, Murat Tanışlı, Kudret Özdaş, and Süleyman Demir \\ Anadolu University, Science Faculty, Department of Physics, 26470 Eskişehir, Turkey \\ Reprint requests to M. T.; E-mail: mtanisli@anadolu.edu.tr
}

Z. Naturforsch. 63a, 15-18 (2008); received July 5, 2007

In this study, after introducing the hyperbolic octonionic (counteroctonion) algebra, which is also
expressed in the sub-algebra of sedenions, and differential operator, Proca-Maxwell equations and
relevant field equations are derived in compact, simpler and elegant forms using hyperbolic octonions.
This formalism demonstrates that Proca-Maxwell equations can be expressed in a single equation.

Key words: Hyperbolic Octonion; Proca Field Equation; Proca-Maxwell Equations.

PACS numbers: 02.10.De, 03.50.De

\section{Introduction}

Today, in addition to usual vector and tensor algebras, many different algebras are used in physics. One of the well-known of these algebras is the complex algebras, which can be extended into 4,8 and 16 dimensions. Some of these algebras are also noncommutative and non-associative.

The complex algebras become indispensable algebraic structures for expressing the quantum theory [1-6] in physics. Quaternions, octonions and 16dimensional sedenions are used for denoting physical events. These algebraical structures play an important role in understanding physical equations and getting compact representations.

There are many publications referring to these algebraical structures directly in their title: The Pauli equation in scale relativity [7], On the reduction of the multidimensional stationary Schrödinger equation to a first-order equation and its relation to the pseudoanalytic function theory [8], Application of bicomplex (quaternion) algebra to fundamental electromagnetism: A lower order alternative to the Helmholtz equation [9], Maxwell's theory on non-commutative space and quaternions [10], Quaternionic formulation of the classical fields [11], Quaternionic quantum mechanics and quantum fields [12], Quaternionic diffusion by potential step [13], A novel solution to Kepler's problem [14], Gravity on octonion algebra [15], Feymann's derivation of Maxwell equations and extra dimensions [16], Eight-dimensional quantum Hall effect and octonions [17], Reformulation of electromagnetism with octonions [18], Octo- nionic strong and weak interactions and their quantum equation [19], Elementary operations [20], Clifford algebraic spinor and the Dirac wave equations [21], Geometric algebra techniques for general relativity [22], The spacetime algebra approach to massive classical electrodynamics with magnetic monopoles [23], Relativistic quantum physics with hyperbolic numbers [24], Dirac equation hyperbolic octonions [25], Gravity and electromagnetism on conic sedenion [26], Hypernumber and relativity [27], Signature of gravity in conic sedenions [28].

The organization of the paper is as follows: Section 2 reveals hyperbolic octonions (counteroctonion) with notations and preliminaries. Proca-Maxwell equations are introduced in Section 3. Section 4 implies the Proca field equation and Proca-Maxwell equations in a single equation using hyperbolic octonions. A summary and perspective of our work are given in the final section.

\section{Hyperbolic (Countercomplex) Octonions}

A hyperbolic octonion, $\mathbb{Q}$, is an 8 -dimensional hypercomplex number and

$$
\begin{aligned}
\mathbb{Q}= & a_{0}+a_{1} \mathbf{e}_{1}+a_{2} \mathbf{e}_{2}+a_{3} \mathbf{e}_{3} \\
& +a_{4} \boldsymbol{\varepsilon}_{4}+a_{5} \boldsymbol{\varepsilon}_{5}+a_{6} \boldsymbol{\varepsilon}_{6}+a_{7} \boldsymbol{\varepsilon}_{7}
\end{aligned}
$$

where $a_{0}, a_{1}, a_{2}, a_{3}, a_{4}, a_{5}, a_{6}, a_{7}$ are real numbers, $\mathbf{e}_{1}$, $\mathbf{e}_{2}, \mathbf{e}_{3}$ are quaternion units, and $\boldsymbol{\varepsilon}_{4}\left(\boldsymbol{\varepsilon}_{4}^{2}=1\right)$ is a counterimaginary unit. Literally in octonionic algebra, the bases of hyperbolic octonions are defined by a fourth 
Table 1. Multiplications of bases of hyperbolic octonions [5].

\begin{tabular}{c|ccccccc}
\hline & $\mathbf{e}_{1}$ & $\mathbf{e}_{2}$ & $\mathbf{e}_{3}$ & $\varepsilon_{4}$ & $\varepsilon_{5}$ & $\varepsilon_{6}$ & $\varepsilon_{7}$ \\
\hline $\mathbf{e}_{1}$ & -1 & $\mathbf{e}_{3}$ & $-\mathbf{e}_{2}$ & $\varepsilon_{5}$ & $\varepsilon_{4}$ & $-\varepsilon_{7}$ & $\varepsilon_{6}$ \\
$\mathbf{e}_{2}$ & $-\mathbf{e}_{3}$ & -1 & $\mathbf{e}_{1}$ & $\varepsilon_{6}$ & $\varepsilon_{7}$ & $\varepsilon_{4}$ & $-\varepsilon_{5}$ \\
$\mathbf{e}_{3}$ & $\mathbf{e}_{2}$ & $-\mathbf{e}_{1}$ & -1 & $\varepsilon_{7}$ & $-\varepsilon_{6}$ & $\varepsilon_{5}$ & $\varepsilon_{4}$ \\
$\varepsilon_{4}$ & $-\varepsilon_{5}$ & $-\varepsilon_{6}$ & $-\varepsilon_{7}$ & 1 & $\mathbf{e}_{1}$ & $\mathbf{e}_{2}$ & $\mathbf{e}_{3}$ \\
$\varepsilon_{5}$ & $-\varepsilon_{4}$ & $-\varepsilon_{7}$ & $\varepsilon_{6}$ & $-\mathbf{e}_{1}$ & 1 & $\mathbf{e}_{3}$ & $-\mathbf{e}_{2}$ \\
$\varepsilon_{6}$ & $\varepsilon_{7}$ & $-\varepsilon_{4}$ & $-\varepsilon_{5}$ & $-\mathbf{e}_{2}$ & $-\mathbf{e}_{3}$ & 1 & $\mathbf{e}_{1}$ \\
$\varepsilon_{7}$ & $-\varepsilon_{6}$ & $\varepsilon_{5}$ & $-\varepsilon_{4}$ & $-\mathbf{e}_{3}$ & $\mathbf{e}_{2}$ & $-\mathbf{e}_{1}$ & 1 \\
\hline
\end{tabular}

unit and quaternionic units as follows:

$$
\begin{aligned}
& \mathbf{e}_{1} \boldsymbol{\varepsilon}_{4}=\boldsymbol{\varepsilon}_{5}, \quad \mathbf{e}_{2} \boldsymbol{\varepsilon}_{4}=\boldsymbol{\varepsilon}_{6}, \quad \mathbf{e}_{3} \boldsymbol{\varepsilon}_{4}=\boldsymbol{\varepsilon}_{7} \\
& \left(\boldsymbol{\varepsilon}_{5}{ }^{2}=\boldsymbol{\varepsilon}_{6}{ }^{2}=\boldsymbol{\varepsilon}_{7}{ }^{2}=1\right) .
\end{aligned}
$$

The bases of hyperbolic octonions have multiplication rules as in Table 1. by

The conjugate of the hyperbolic octonion $\mathbb{Q}$ is given

$$
\begin{aligned}
\overline{\mathbb{Q}}= & a_{0}-a_{1} \mathbf{e}_{1}-a_{2} \mathbf{e}_{2}-a_{3} \mathbf{e}_{3} \\
& -a_{4} \boldsymbol{\varepsilon}_{4}-a_{5} \boldsymbol{\varepsilon}_{5}-a_{6} \boldsymbol{\varepsilon}_{6}-a_{7} \boldsymbol{\varepsilon}_{7},
\end{aligned}
$$

just as for the octonions. The quadratic form (or square norm) of $\mathbb{Q}$ is

$$
\begin{aligned}
N(\mathbb{Q}) & =\mathbb{Q} \overline{\mathbb{Q}} \\
& =a_{0}{ }^{2}+a_{1}{ }^{2}+a_{2}{ }^{2}+a_{3}{ }^{2}-a_{4}{ }^{2}-a_{5}{ }^{2}-a_{6}{ }^{2}-a_{7}{ }^{2} .
\end{aligned}
$$

This norm is an usual pseudo-Euclidean norm on $R^{4,4}$. The norm is isotropic, meaning that there are nonzero $\mathbb{Q}$ for which $N(\mathbb{Q})=0$. An element $\mathbb{Q}$ has on inverse, $\mathbb{Q}^{-1}$, if and only $N(\mathbb{Q}) \neq 0$. In this case, the inverse of $\mathbb{Q}$ is given by

$$
\mathbb{Q}^{-1}=\frac{\overline{\mathbb{Q}}}{N(\mathbb{Q})} .
$$

It is easy to see that the multiplication of hyperbolic octonions satisfies

$$
\mathrm{AB}=-\mathrm{BA}, \quad \mathrm{A}, \mathrm{B}=\mathbf{e}_{i}, \boldsymbol{\varepsilon}_{i+4}, \boldsymbol{\varepsilon}_{4}, \quad i=1,2,3,
$$

namely, this algebra is anti-commute and

$$
\left(\boldsymbol{\varepsilon}_{m} \mathbf{e}_{n}\right) \boldsymbol{\varepsilon}_{p}=-\boldsymbol{\varepsilon}_{m}\left(\mathbf{e}_{n} \boldsymbol{\varepsilon}_{p}\right) \quad(m \neq n, n \neq p, p \neq m) .
$$

\section{Proca-Maxwell Equations}

The well-known Maxwell equations and Maxwell's Lagrangian are based on the hypothesis that the photon has zero mass. But it is known that the Lagrangian can be modified by adding a mass term. At the end, the Lagrangian is known as Proca's Lagrangian. In the CGS unit system, Proca's Lagrangian is given by

$$
\mathcal{L}=-\frac{1}{16 \pi} \mathcal{F}_{\mu v} \mathcal{F}^{\mu v}+\frac{m_{\gamma}^{2}}{8 \pi} \mathcal{A}_{\mu} \mathcal{A}^{\mu}-\frac{1}{c} \mathcal{J}_{\mu} \mathcal{A}^{\mu}
$$

where $m_{\gamma}=\frac{\omega}{c}$ is the inverse of the Compton wavelength associated with the photon mass, $\mathcal{J}_{\mu}$ is the fourcurrent $(\mathcal{J} \equiv \rho,-\vec{j}), \mathcal{A}^{\mu}$ is the four-vector potential $\left(\mathcal{A} \equiv A_{0}, \vec{A}\right), \mathcal{F}_{\mu v}=\partial_{\mu} \mathcal{A}_{v}-\partial_{\nu} \mathcal{A}_{\mu}$ denotes the electromagnetic field tensor. The Euler-Lagrange equation is

$$
\frac{\partial \mathcal{L}}{\partial \mathcal{A}_{\mu}}-\partial_{\nu}\left(\frac{\partial \mathcal{L}}{\partial\left(\partial_{\nu} \mathcal{A}_{\mu}\right)}\right)=0 .
$$

Then, the Proca equation is defined as

$$
\partial_{\mu} \mathcal{F}^{\mu v}+m_{\gamma}^{2} \mathcal{A}^{v}=\frac{4 \pi}{c} J^{v}
$$

By using the Lorentz-gauge condition

$$
\partial_{\mu} \mathcal{A}^{\mu}=0
$$

and in terms of the vector potentials, (3) can be written as

$$
\left(\square+m_{\gamma}\right) \mathcal{A}_{\mu}=\frac{4 \pi}{c} J_{\mu} .
$$

Thus, Proca-Maxwell equations are obtained in the vectorial formalism as [23, 29, 30]

$$
\begin{aligned}
& \vec{\nabla} \cdot \vec{E}=4 \pi \rho-m_{\gamma}^{2} A_{0}, \\
& \vec{\nabla} \times \vec{E}=-\frac{1}{c} \frac{\partial \vec{B}}{\partial t}, \\
& \vec{\nabla} \cdot \vec{B}=0, \\
& \vec{\nabla} \times \vec{B}=\frac{4 \pi}{c} \vec{J}+\frac{1}{c} \frac{\partial \vec{E}}{\partial t}-m_{\gamma}^{2} \vec{A},
\end{aligned}
$$

These equations open the new ways for investigations in theoretical and experimental physics.

\section{Hyperbolic Octonionic Proca Field Equation and Proca-Maxwell Equations}

Before the Proca field equation is given by using hyperbolic octonionics, the hyperbolic octonionic differential operator, $\square$, will be defined as

$$
\square=-\frac{1}{c} \frac{\partial}{\partial t}+\frac{\partial}{\partial x} \boldsymbol{\varepsilon}_{5}+\frac{\partial}{\partial y} \boldsymbol{\varepsilon}_{6}+\frac{\partial}{\partial z} \boldsymbol{\varepsilon}_{7},
$$


and its conjugate is

$$
\bar{\square}=-\frac{1}{c} \frac{\partial}{\partial t}-\frac{\partial}{\partial x} \boldsymbol{\varepsilon}_{5}-\frac{\partial}{\partial y} \boldsymbol{\varepsilon}_{6}-\frac{\partial}{\partial z} \boldsymbol{\varepsilon}_{7} .
$$

Hence, the d'Alambert operator can be written as

$$
\square \bar{\square}=\frac{1}{c^{2}} \frac{\partial^{2}}{\partial t^{2}}-\Delta,
$$

with the Laplacian

$$
\Delta=\frac{\partial^{2}}{\partial x^{2}}+\frac{\partial^{2}}{\partial y^{2}}+\frac{\partial^{2}}{\partial z^{2}} .
$$

Using the Dirac equation, the hyperbolic octonionic field can be expressed as [24]

$$
\begin{aligned}
\psi=( & \psi_{4}+\psi_{1} \mathbf{e}_{1}+\psi_{2} \mathbf{e}_{2}+\psi_{3} \mathbf{e}_{3} \\
& \left.-\psi_{4}{ }^{\prime} \boldsymbol{\varepsilon}_{4}-\psi_{1}{ }^{\prime} \boldsymbol{\varepsilon}_{5}-\psi_{2}{ }^{\prime} \boldsymbol{\varepsilon}_{6}-\psi_{3}{ }^{\prime} \boldsymbol{\varepsilon}_{7}\right) .
\end{aligned}
$$

If the general hyperbolic octonionic potential and the hyperbolic octonionic source are defined as

$$
\begin{gathered}
\mathbb{P}=\left(\varphi_{2}+A_{x} \mathbf{e}_{1}+A_{y} \mathbf{e}_{2}+A_{z} \mathbf{e}_{3}\right. \\
\left.+\varphi_{1} \boldsymbol{\varepsilon}_{4}-A_{x}{ }^{\prime} \boldsymbol{\varepsilon}_{5}-A_{y}{ }^{\prime} \boldsymbol{\varepsilon}_{6}-A_{z}{ }^{\prime} \boldsymbol{\varepsilon}_{7}\right), \\
\mathbb{J}=\frac{4 \pi}{c}\left(c \rho_{2}+J_{x} \mathbf{e}_{1}+J_{y} \mathbf{e}_{2}+J_{z} \mathbf{e}_{3}\right. \\
\left.\quad+c \rho_{1} \boldsymbol{\varepsilon}_{4}-J_{x}{ }^{\prime} \boldsymbol{\varepsilon}_{5}-J_{y}{ }^{\prime} \boldsymbol{\varepsilon}_{6}-J_{z}{ }^{\prime} \boldsymbol{\varepsilon}_{7}\right),
\end{gathered}
$$

the general Proca field equation can be written by using hyperbolic octonions:

$$
\square \psi+k_{0}^{2} \mathbb{P}=\mathbb{J} .
$$

It is also obvious that

$$
\square \mathbb{P}=\psi .
$$

In (17), $k_{0}$ is a physical constant, which is formed by choosing a potential.

Equation (17) can be clearly expressed by the following equations:

$$
\begin{aligned}
& -\frac{1}{c} \frac{\partial \psi_{4}}{\partial t}+\frac{\partial \psi_{1}{ }^{\prime}}{\partial t}+\frac{\partial \psi_{2}{ }^{\prime}}{\partial y}+\frac{\partial \psi_{3}{ }^{\prime}}{\partial z}+k_{0}^{2} \varphi_{2}=4 \pi \rho_{2} \\
& -\frac{1}{c} \frac{\partial \psi_{1}}{\partial t}-\frac{\partial \psi_{4}{ }^{\prime}}{\partial x}+\frac{\partial \psi_{3}{ }^{\prime}}{\partial y}-\frac{\partial \psi_{2}{ }^{\prime}}{\partial z}+k_{0}^{2} A_{x}=\frac{4 \pi}{c} J_{x} \\
& -\frac{1}{c} \frac{\partial \psi_{2}}{\partial t}-\frac{\partial \psi_{4}{ }^{\prime}}{\partial y}-\frac{\partial \psi_{3}{ }^{\prime}}{\partial x}+\frac{\partial \psi_{1}{ }^{\prime}}{\partial z}+k_{0} A_{x}=\frac{4 \pi}{c} J_{y}
\end{aligned}
$$

$$
\begin{gathered}
-\frac{1}{c} \frac{\partial \psi_{3}}{\partial t}-\frac{\partial \psi_{4}{ }^{\prime}}{\partial z}+\frac{\partial \psi_{2}{ }^{\prime}}{\partial x}-\frac{\partial \psi_{1}{ }^{\prime}}{\partial y}+k_{0} A_{x}=\frac{4 \pi}{c} J_{z} \\
\frac{1}{c} \frac{\partial \psi_{4}{ }^{\prime}}{\partial t}+\frac{\partial \psi_{1}}{\partial y}+\frac{\partial \psi_{2}}{\partial x}+\frac{\partial \psi_{3}}{\partial z}+k_{0} \varphi_{1}=4 \pi \rho_{1}, \\
\frac{1}{c} \frac{\partial \psi_{1}{ }^{\prime}}{\partial t}-\frac{\partial \psi_{4}}{\partial x}+\frac{\partial \psi_{3}}{\partial y}-\frac{\partial \psi_{2}}{\partial z}-k_{0} A_{x}{ }^{\prime}=-\frac{4 \pi}{c} J_{x}{ }^{\prime}, \\
\frac{1}{c} \frac{\partial \psi_{2}{ }^{\prime}}{\partial t}-\frac{\partial \psi_{4}}{\partial y}-\frac{\partial \psi_{3}}{\partial x}+\frac{\partial \psi_{1}}{\partial z}-k_{0} A_{y}{ }^{\prime}=-\frac{4 \pi}{c} J_{y}{ }^{\prime} \\
\frac{1}{c} \frac{\partial \psi_{3}{ }^{\prime}}{\partial t}-\frac{\partial \psi_{4}}{\partial z}+\frac{\partial \psi_{2}}{\partial x}-\frac{\partial \psi_{1}}{\partial y}-k_{0} A_{z}{ }^{\prime}=-\frac{4 \pi}{c} J_{z}
\end{gathered}
$$

In order to define Proca-Maxwell equations, the hyperbolic octonionic four-potential and hyperbolic octonionic four-current are chosen as

$$
\begin{aligned}
& \mathbb{A}=A_{x} \mathbf{e}_{1}+A_{y} \mathbf{e}_{2}+A_{z} \mathbf{e}_{3}+\phi \boldsymbol{\varepsilon}_{4}, \\
& \mathbb{J}=\frac{4 \pi}{c}\left(J_{x} \mathbf{e}_{1}+J_{y} \mathbf{e}_{2}+J_{z} \mathbf{e}_{3}+c \rho\right) .
\end{aligned}
$$

Then the hyperbolic octonionic electromagnetic field is specified:

$$
\square \mathbb{A}=\mathbb{F} .
$$

It is possible to clearly write (22) in terms of the components:

$$
\begin{aligned}
\square \mathbb{A}= & \left(-\frac{\partial A_{x}}{\partial t}-\frac{\partial \phi}{\partial x}\right) \mathbf{e}_{1}+\left(-\frac{\partial A_{y}}{\partial t}-\frac{\partial \phi}{\partial y}\right) \mathbf{e}_{2} \\
& +\left(-\frac{\partial A_{z}}{\partial t}-\frac{\partial \phi}{\partial z}\right) \mathbf{e}_{3} \\
& +\left(-\frac{\partial \phi}{\partial t}-\frac{\partial A_{x}}{\partial x}-\frac{\partial A_{y}}{\partial y}-\frac{\partial A_{z}}{\partial z}\right) \boldsymbol{\varepsilon}_{4} \\
& +\left(-\frac{\partial A_{z}}{\partial y}+\frac{\partial A_{y}}{\partial z}\right) \boldsymbol{\varepsilon}_{5} \\
& +\left(-\frac{\partial A_{z}}{\partial x}+\frac{\partial A_{x}}{\partial z}\right) \boldsymbol{\varepsilon}_{6}+\left(\frac{\partial A_{x}}{\partial y}-\frac{\partial A_{y}}{\partial x}\right) \boldsymbol{\varepsilon}_{7}
\end{aligned}
$$

in which

$$
-\frac{\partial \phi}{\partial t}-\vec{\nabla} \cdot \vec{A}=0
$$

is accepted as the usual Lorentzian condition. Then, the electromagnetic field is

$$
\mathbb{F}=E_{x} \mathbf{e}_{1}+E_{y} \mathbf{e}_{2}+E_{z} \mathbf{e}_{3}-B_{x} \boldsymbol{\varepsilon}_{5}-B_{y} \boldsymbol{\varepsilon}_{6}-B_{z} \boldsymbol{\varepsilon}_{7}
$$


Consequently, the Proca-Maxwell equation is

$$
\bar{\square} \mathbb{F}+m_{\gamma}^{2} \mathbb{A}=\mathbb{J},
$$

and (25) is described in terms of components by

$$
\begin{aligned}
& \frac{\partial B_{x}}{\partial x}+\frac{\partial B_{y}}{\partial y}+\frac{\partial B_{z}}{\partial z}=0 \\
& -\frac{1}{c} \frac{\partial E_{x}}{\partial t}+\frac{\partial B_{z}}{\partial y}-\frac{\partial B_{y}}{\partial z}+m_{\gamma}^{2} A_{x}=\frac{4 \pi}{c} J_{x} \\
& -\frac{1}{c} \frac{\partial E_{y}}{\partial t}-\frac{\partial B_{z}}{\partial x}+\frac{\partial B_{x}}{\partial z}+m_{\gamma}^{2} A_{y}=\frac{4 \pi}{c} J_{y}, \\
& -\frac{1}{c} \frac{\partial E_{z}}{\partial t}+\frac{\partial B_{y}}{\partial x}-\frac{\partial B_{x}}{\partial y}+m_{\gamma}^{2} A_{z}=\frac{4 \pi}{c} J_{z} \\
& \frac{\partial E_{x}}{\partial x}+\frac{\partial E_{y}}{\partial y}+\frac{\partial E_{z}}{\partial z}+m_{\gamma}^{2} \phi=4 \pi \rho \\
& \frac{1}{c} \frac{\partial B_{x}}{\partial t}+\frac{\partial E_{z}}{\partial y}-\frac{\partial E_{y}}{\partial z}=0 \\
& \frac{1}{c} \frac{\partial B_{y}}{\partial t}-\frac{\partial E_{z}}{\partial x}+\frac{\partial E_{x}}{\partial z}=0 \\
& \frac{1}{c} \frac{\partial B_{z}}{\partial t}+\frac{\partial E_{y}}{\partial x}-\frac{\partial E_{x}}{\partial y}=0 .
\end{aligned}
$$

[1] C. Musés, Appl. Math. Comput. 3, 211 (1976).

[2] C. Musés, Appl. Math. Comput. 6, 63 (1980).

[3] C. Musés, Appl. Math. Comput. 4, 45 (1978).

[4] C. Musés, Appl. Math. Comput. 6, 25 (1994).

[5] K. Carmondy, Appl. Math. Comput. 88, 47 (1988).

[6] K. Carmondy, Appl. Math. Comput. 84, 27 (1997).

[7] M. N. Célérier and L. Nottale, arXiv: quant$\mathrm{ph} / 0609107$.

[8] H. T. Anastassiu, P.E. Atlamazoğlu, and D. I. Kaklamani, IEEE Trans. Antennas Propag. 51, 2130 (2003).

[9] V. V. Kravchenko, J. Phys. A: Math. Gen. 38, 851 (2005).

[10] S. I. Kruglov, arXiv: hep-th/0110059.

[11] V. Majernik, Adv. Appl. Cliff. Alg. 9, 119 (1999).

[12] L. A. Adler, Quaternionic Quantum Mechanics and Quantum Fields, Oxford University Press, New York 1995.

[13] S. D. Leo and G. C. Ducati, J. Math. Phys. 47, 102104 (2006).

[14] J. Vrbik, Eur. J. Phys. 24, 575 (2003).

[15] V. Dorofoev, arXiv: gr-qc/0604024.

[16] Z. K. Silagadze, Ann. Fond. Louis Broglie 27, 241 (2002) (arXiv: hep-ph/0106235).

[17] B. A. Bernevig, J. Hu, N. Toumbas, and S. C. Zhang, Phys. Rev. Lett. 91, 236803 (2003).
In the above equations, if $m_{\gamma}=0,(25)$ is equal to the well-known Maxwell equations.

\section{Conclusions}

In this study, the generalized Maxwell equations in quantum field theory are formulated by using hyperbolic octonions. It is shown that a multivectoral equation is enough to describe the theory. The obtained results are the same as usual Proca-Maxwell equations, and these equations have also easy and compact representations. This study proofs the claims made by Musés [4] and Carmondy [6], in which hyperbolic octonions can be used in quantum theory. As it is known, octonions are used for representations in classical electromagnetic and Dirac equations [31,32]. Thus hyperbolic octonions, which have the same mathematical analogy with octonions and split octonions, will be used for non-associative quantum mechanics [33]. Furthermore, hyperbolic octonions will be useful tools for obtaining the Proca-Maxwell equations with magnetic monopole and gravitoelectromagnetism equations of Einstein's field equation in the weak field approach.

[18] T. Tolan, K. Özdaş, and M. Tanışlı, IL Nuovo Cimento 121(B), 43 (2006).

[19] Z. Weng, arXiv: physics/0612102.

[20] J. Baugh, A. Galiautdinov, D. Ritz Finkelstein, and M. Shiri-Garakani, arXiv: quant-ph/0411213.

[21] Y. Xuegang, Z. Shuna, and H. Qiunan, Adv. Appl. Clifford Alg. 11, 27 (2001).

[22] M. R. Francis and A. Kosowsky, Ann. Phys. 311, 459 (2004).

[23] C. Cafaro and S. A. Ali, Adv. Appl. Clifford Alg. 17, 23 (2007).

[24] S. Ulrych, Phys. Lett. B 25, 313 (2005).

[25] J. Köplinger, Appl. Math. Comput. 182, 443 (2006).

[26] J. Köplinger, Appl. Math. Comput. 188, 948 (2007).

[27] J. Köplinger, Appl. Math. Comput. 188, 954 (2007).

[28] J. Köplinger, Appl. Math. Comput. 188, 942 (2007).

[29] J. D. Jackson, Classical Electrodynamics, John Wiley \& Sons, New York 1962, pp. 598-601.

[30] J. Luo, C. G. Shao, Z.Z Liu, and Z. K. Hu, Phys. Lett. A 270, 288 (2000) (arXiv:gr-qc/0607004).

[31] M. Gogberashvili, Octonionic Version of Dirac Equations, (arXiv: hep-th/0505101).

[32] M. Gogberashvili, J. Phys. A: Math Gen. 39, 7099 (2006) (arXiv: hep-th/0512258).

[33] V. Dzhunushaliev, Found. Phys. Lett. 19, 157 (2006) (arXiv: hep-th/0502216). 